\title{
Research Paper: Differential Dynamics of the Levels of Low Molecular Weight DNA Fragments in the Plasma of Patients With Ischemic and Hemorrhagic Strokes
}

\author{
Irina Vasilyeva $^{1,2^{*}}$ (D), Vladimir Bespalov ${ }^{1,2}$ (D), Ancha Baranova ${ }^{3,4,5}$ (D), Igor Voznyuk ${ }^{6}$, Denis Baranenko ${ }^{6}$ (D)
}

1. Laboratory of Cancer Chemoprevention and Oncopharmacology, N.N.Petrov National Medical Research Center of Oncology, St.-Petersburg, Russia. 2. International Research Center 'Biotechnologies of the Third Millennium'ITMO University, St.-Petersburg, Russia.

3. Department of George, School of Systemic Biology, George Mason University, Fairfax, VA, USA.

4. Laboratory of Functional Genomics, Research Center for Medical Genetics, Moscow, Russia.

5. Scientific Council, Atlas Biomed Group, Moscow, Russia.

6. Department of Acute Cerebrovascular Pathology and Emergency Neurology, Saint-Petersburg I.I. Dzhanelidze Research Institute for Emergency

Medicine, St.-Petersburg, Russia.

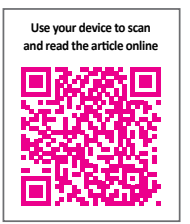

Citration: Vasilyeva, I., Bespalov, V., Baranova, A., Voznyuk, I., \& Baranenko, D. (2020). Differential Dynamics of the Levels of Low Molecular Weight DNA Fragments in the Plasma of Patients With Ischemic and Hemorrhagic Strokes. Basic and Clinical Neuroscience, 11(6), 805-810. http://dx.doi.org/10.32598/bcn.11.6.1639.1

\section{(c) (1) (s)}

Article info:

Received: 19 Dec 2018

First Revision: 10 Jan 2019

Accepted: 16 Feb 2019

Available Online: 01 Nov 2020

Keywords:

Acute cerebrovascular accident, Cerebral ischemia, Hemorrhagic stroke, Cell-free DNA, Apoptosis

\section{A B S T RA C T}

Introduction: To evaluate Low-Molecular-weight (LMW) DNA as a possible prognostic biomarker in acute ischemic and hemorrhagic stroke.

Methods: LMW DNA samples were isolated from plasma and cerebrospinal fluid by phenol deproteinization, analyzed by gradient polyacrylamide electrophoresis and quantified by spectrophotometry.

Results: Two common types of stroke, i.e. ischemic and hemorrhagic, differ by the temporal dynamics of cell-free DNA (cfDNA) accumulation. In hemorrhagic stroke, an initial increase in LMW DNA levels, most likely reflects an extent of the tissue damage, while in ischemic patients, the LMW DNA levels increase in parallel with the damage caused by hypoxia and subsequent compensatory reperfusion.

Conclusion: These time-course data specify optimal assessment windows with maximum differentiating power for stroke outcomes: $24-48$ hours post-event for ischemic stroke, and as close as possible to the moment of hospital admission for hemorrhagic stroke. These data also indicate the role of apoptosis in the formation of ischemic focus.

\section{* Corresponding Author:}

Irina Vasilyeva, PhD.

Address: Laboratory of Cancer Chemoprevention and Oncopharmacology, N.N.Petrov National Medical Research Center of Oncology, St.-Petersburg, Russia. Tel: + 7 (921) 903441

E-mail: iravasilyeva@hotmail.com 


\section{Highlights}

- Ischemic and hemorrhagic stroke differ by the temporal dynamics of cell-free DNA (cfDNA) accumulation.

- In hemorrhagic stroke, an initial increase in cfDNA, most likely reflects an extent of the tissue damage

- In ischemic patients, the apoptotic cfDNA increase in parallel with the damage caused by hypoxia and subsequent compensatory reperfusion.

- An optimal assessment windows with maximum differentiating power for stroke outcomes is 24-48 hours postevent for ischemic stroke, and as close as possible to the moment of hospital admission for hemorrhagic stroke.

\section{Plain Language Summary}

Acute cerebrovascular accidents are a prominent cause of death worldwide and are the leading cause of long-term disability. Stroke is a phenotypically, various disease. The outcome of acute cerebrovascular accidents is largely determined by the timeliness and adequacy of treatment, which is fundamentally different in patients with ischemic and hemorrhagic stroke. Cell-free DNA is released from apoptotic and necrotic cells, and its fragments circulate in the blood until cleared by DNA hydrolyzing enzymes. In plasma, cell-free DNA is represented by fragments of various sizes, with the most common fragment length corresponding to the nucleosome's size and reflecting its apoptotic origin. LmwDNA samples were isolated from plasma and cerebrospinal fluid by phenol deproteinization; they were analyzed by gradient polyacrylamide electrophoresis and were quantified by spectrophotometry. Two common types of stroke, ischemic and hemorrhagic, differ by the temporal dynamics of cell-free DNA accumulation. In hemorrhagic stroke, an initial increase in lmwDNA levels most likely reflects an extent of the tissue damage. In contrast, in ischemic patients, the lmwDNA levels increase in parallel with the damage caused by hypoxia and subsequent compensatory reperfusion. These time-course data specify optimal assessment windows with maximum differentiating power for stroke outcomes: 24-48 hours post-event for ischemic stroke, and as close as possible to the moment of hospital admission for hemorrhagic stroke. These data also indicate the role of apoptosis in the formation of ischemic focus.

\section{Introduction}

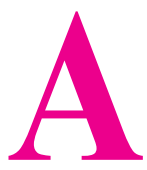

cute ischemia of brain tissue resulting in a stroke is a prominent cause of death worldwide, with more than 15 million cases diagnosed annually (Mackay \& Mensah, 2004). In the USA alone, one stroke occurs in almost 800000 people every year and is the main cause of long-term disability (Go et al., 2013).

Stroke is a phenotypically diverse disease. Most beats (87\%) as a result of blood vessel clotting, cutting off blood flow to a part of the brain and is therefore well diagnosed. The rest of the strokes are due to hemorrhage. On top of that, stroke may affect widely disparate areas of the brain, thus providing for the diversity of symptoms. Tailoring the therapy during the acute, recovery, and secondary prevention phases of post-stroke treatment requires the development and validation of the biomarkers suitable for early evaluation of the extent of pathophysiological processes associated with stroke.
Energy metabolism in the brain is primarily determined by aerobic glycolysis, and the glucose content in the brain is depleted after 5-6 minutes, leading to disruption of the mitochondrial chain and the decrease in ATP (Choi \& Pile-Spellman, 2018). Oxidative stress and associated oxidative damage are the main causes of cell damage and cell death during ischemia. Reactive oxygen species (superoxide radical anion, hydroxyl radical, singlet oxygen, and hydrogen peroxide) damage cellular targets such as DNA, proteins, and lipids. After ischemia, reperfusion can cause a sequential chain of pathophysiological cascades, including massive intracellular $\mathrm{Ca}^{2+}$ release, neutrophil recruitment, acute inflammatory reactions, and the formation of free radicals, which, in turn, cause apoptotic or necrotic cell death (Amani et al., 2017).

After its release from apoptotic and necrotic cells, cellfree DNA (cfDNA) fragments circulate in the blood until cleared by DNA hydrolyzing enzymes (Mittra, Nair, \& Mishra, 2012). Additionally, at least some of the released DNA fragments got included in various supramolecular complexes, where they remain stable for a long time. If 
the cell death toll exceeds the capacity of the clearance mechanisms, the fraction of cell-free DNA increases in its abundance (Peters \& Pretorius, 2011). The animal model demonstrated that this is, indeed, the case for acute ischemic events (Boyko et al., 2011).

From a mechanistic perspective, the greater the size of an area affected by stroke, the more cell death there will be, and, consequently, the more cfDNA release. There is also evidence that cfDNA released from the dying cell may serve as an activation or stress signal for immune and other cells, resulting in pro-inflammatory cytokine responses (Hefeneider et al., 1992; Glebova, Veiko, Kostyuk, Izhevskaya, \& Baranova, 2015; Ermakov et al., 2013). In plasma, cfDNA is represented by fragments of various sizes, with the most common fragment length corresponding to the size of the nucleosome and reflecting its apoptotic origin. In the case of necrosis, relatively long fragments of DNA, about $10000 \mathrm{bp}$ in length are produced (Zhivotovsky \& Orrenius, 2001).

Previous studies showed that the levels of cfDNA are predictive for stroke outcomes when measured as late as 6 weeks post-event (Rainer et al., 2003; Lam, Rainer, Wong, Lam, \& Lo, 2006; Geiger et al., 2006) with larger levels pointing toward possible mortality, or to the prominence of ischemic sequelae (Vasilyeva, Ivtchik, \& Voznyuk, 2010). On the other hand, a gradual decrease in the levels of cfDNA indicates an improvement and may be used as a biomarker of recovery (Vasilyeva, Ivtchik, \& Voznyuk, 2010). Here we present the results of a comparative study of LMW cfDNA levels in plasma and $\mathrm{CSF}$ of the patients with hemorrhagic and ischemic brain lesions at early post-stroke time points.

\section{Methods}

\subsection{Settings and participants}

The patients with acute stroke $(n=54)$ aged $46-50$ years were enrolled upon admission to the I.I. Djanelidze State Research Institute for Emergency Hospital (St.Petersburg, Russia) between January 2013 and November 2016, and investigated prospectively. Acute ischemic cerebrovascular accident, as a sudden loss of focal cerebral function, persisted for 24 hours and was diagnosed according to clinical presentation. All participants had no clinical evidence of prior stroke. Five of them were diagnosed with hemorrhagic stroke, and 46 with ischemic stroke. For each patient with hemorrhagic stroke, age, and gender matching patient was selected from the group with ischemic stroke and underwent DNA testing in plasma.
The control subjects $(n=23)$ at age of 34-73 were enrolled at the time of the annual check-up. None of the controls had any evidence of prior strokes or other major healthrelated events within a year before the study. Donors were divided into 3 age groups: $34-44$ years $(n=7), 46-67$ years old, corresponding to the age of patients $(n=6)$, and 60 74 years old $(n=4)$. After venipuncture, $3 \mathrm{~mL}$ samples of blood were collected in EDTA (Ethylenediaminetetraacetic acid) containing Vacutainer tubes (BD, USA)

Post-ischemic stroke patients $(n=42)$ were enrolled at the time of admission and underwent collection of Cerebrospinal Fluids (CSF) as part of their assessment. Leftover CSF samples available for DNA analysis included ones collected at a timeframe of $2-24$ hours $(n=5), 2-7$ days $(n=12), 7-21$ days $(n=8)$, more than 21 days $(n=17)$.

\subsection{Blood Sampling and Assessment of Plasma DNA}

For all patients enrolled in the ischemic/hemorrhagic comparison study, blood samples were collected at different times after an acute onset of the symptoms: 3,6 , $12,24,48$, and 72 hours. Samples of $3 \mathrm{~mL}$ of peripheral venous blood were collected into Vacutainers containing EDTA. Plasma was separated by spinning at $810 \times \mathrm{g}$ for $20 \mathrm{~min}$ at $4 \mathrm{o} \mathrm{C}$ in a bucket rotor. To ensure complete removal of cells, the collected plasma samples were then centrifuged twice at $2200 \times \mathrm{g}$ for $10 \mathrm{~min}$. Nucleic acids were isolated from samples of $200 \mu \mathrm{L}$ of plasma using the QIAamp Blood Kit (Qiagen, Frederick, MD) according to the manufacturer's recommendations "blood and body fluid protocol". Extracted DNA samples were treated by RNAse, analyzed by electrophoresis in polyacrylamide gradient $(2.16 \%)$ in TRIS buffer $0.04 \mathrm{M}$ (acetate, $\mathrm{pH}$ 7.7) with EDTA 0.1 M. LMW fractions of DNA were extracted from gels using QIAquick ${ }^{\circledR}$ Gel Extraction Kits (Qiagen, USA) and evaluated quantitatively by NanoDrop spectrophotometer (ND-1000; Thermo Fisher Scientific, USA).

\subsection{Statistical analysis}

The obtained data were presented as Mean \pm standard deviation or median. The Student's t-test was used for univariate analysis, and the Chi-squared test or Fisher exact-test to compare categorical variables. All statistical analyses were conducted using GraphPad Prism6 (GraphPad Software, USA). 


\section{Results}

In the plasma of 17 healthy donors, the Mean \pm SD levels of LMW fraction of cfDNA was $30.2 \pm 6.6 \mathrm{ng} / \mathrm{mL}$. However, when the donors' group was trimmed down to 5 to match the age distribution in stroke patients (36-67 years old), the Mean \pm SD level of LMW fraction of cfDNA was 20.16 (7.70) ng/mL (these differences had not reached significance; supplementary (Table 1). In group comparisons with stroke patients, the levels of LWM DNA in plasma of an entire cohort of healthy patients were considered.

The levels of LMW fraction of cfDNA in plasma were traced in plasma of 8 patients with ischemic and 5 patients with hemorrhagic stroke (Figure 1). In hemorrhagic stroke, the mean levels of cfDNA were significantly higher than normal at inception through post-stroke day 2, reaching $72.8(17.6) \mathrm{ng} / \mathrm{mL}$ after $3 \mathrm{~h}$ and 61.8 (16.5) $\mathrm{ng} / \mathrm{mL}$ at 6 hours $(\mathrm{P}<0.015)$, decreasing after $24 \mathrm{~h}$ to 28.0 (6.9) $\mathrm{ng} / \mathrm{mL}$ and falling to baseline at $48 \mathrm{~h}$ and to $72 \mathrm{~h}$ (Figure 1). In case of ischemic stroke, significant plasma cfDNA LMW concentrations were detected at inception, 3 hours post-event, when they were 40.2 (7.8) ng/mL, more than twice of that amount in healthy controls, followed by a significant increase to $50.2(5.1) \mathrm{ng} / \mathrm{mL}$ after $24 \mathrm{~h}$ and 54.7 (10.1) ng/mL after $48 \mathrm{~h}(\mathrm{P}<0.02)$. Across the next 72 hours, these levels gradually return to baseline.

In patients with ischemic stroke, detectable amounts of LMW cfDNA were also detected in spinal fluid samples (Table 1) collected approximately 24 hours post-event $(71.9 \pm 16.9 \mathrm{ng} / \mathrm{mL})$, while the samples collected shortly after the stroke and or at later time points were cfDNA negative.

\section{Discussion}

The diagnosis of stroke typically relies on a combination of the clinical timeline of reported or observed events with the results of neurological examination and neuroimaging. The rapid workflow of acute stroke therapy limits the use of laboratory biomarkers for an actual diagnosis. Nevertheless, the phases of recovery and prevention of secondary strokes present a considerable window of opportunity for introducing biomarker-based

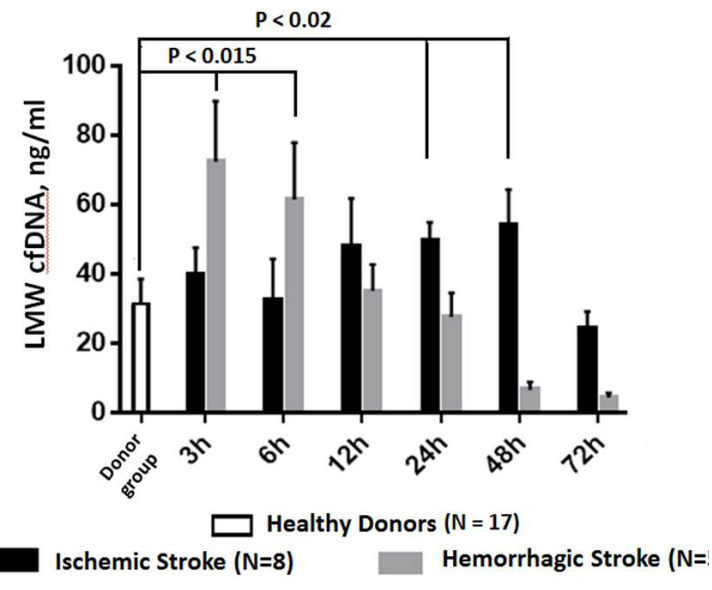

NEUR SCIENCE

Figure 1. LMW DNA levels in the plasma of patients with ischemic or hemorrhagic stroke

Individual LMW cfDNA levels in the plasma of healthy donors.

subtyping (Dieplinger et al., 2017; Bustamante, Simats, Vilar-Bergua, Garcia-Berrocoso, \& Montaner, 2016). This subtyping may be performed during an assessment of the patient's progress at a point-of-care and will provide for the stratification of risks. The use of plasma cfDNA as an integrative biomarker of body stress and cellular apoptosis may serve as a window into the extent of the damage sustained during the stroke.

To develop cfDNA as a biomarker, its plasma levels should be profiled at various post-event time points and, eventually, correlated with outcomes. Stroke-associated elevation of cfDNA levels has been noted previously (Rainer et al., 2003, Lam, Rainer, Wong, Lam, \& Lo, 2006; Geiger et al., 2006; O'Connel et al., 2017; Tsai et al., 2011; Dilek et al., 2013). The current study differs from the ones cited above in two important aspects. It specifically profiled the LMW fraction of DNA released from the cells dying in course of apoptosis, but not from the necrotic cells. Importantly, this approach also emphasizes the evaluation of the short fragments generated in apoptosis rather than fragments of nucleosome-free mtDNA, characterized by more uniform, peakless size distribution.

Table 1. LMW cfDNA concentrations in CSF samples collected at various time points post-ischemic stroke

\begin{tabular}{|c|c|c|c|c|c|}
\hline $\begin{array}{l}\text { Time Frame of CSF Collec- } \\
\text { tion }\end{array}$ & $2 h(n=1)$ & $24 h(n=4)$ & $2-7 d(n=12)$ & 2-3 Weeks ( $n=8)$ & $\begin{array}{l}\text { More Than } 3 \text { Weeks } \\
(n=17)\end{array}$ \\
\hline Concentrations of LMW DNA & Not detected & $71.9 \pm 16.9 \mathrm{ng} / \mathrm{mL}$ & Not detected & Not detected & Not detected \\
\hline
\end{tabular}


Our observational study on LMW cfDNA levels in the plasma of stroke patients showed that two common types of stroke, i.e. ischemic and hemorrhagic, differ by the temporal dynamics of cfDNA accumulation. Sample sizes in both ischemic and hemorrhagic stroke groups were enough to reach a power of 0.8 for alpha significance levels set at 0.05 . In hemorrhagic stroke, the initial increase in LMW cfDNA levels, most likely reflects the extent of the tissue damage, while in ischemic patients, the LMW cfDNA levels increase in parallel with the damage caused by hypoxia and subsequent compensatory reperfusion. These time-course data augment previously reported findings concerning post-stroke plasma cfDNA levels (O'Connel et al., 2017; Tsai et al., 2011; Dilek et al., 2013) by specifying an assessment window with maximum differentiating power for stroke outcomes i.e. 24-48 hours post-event for ischemic stroke, and as close as possible to the moment of hospital admission for hemorrhagic stroke.

In our study, we also enrolled post-stroke patients into the protocol that allowed us to collect and study the leftovers of Cerebrospinal Fluid (CSF) collected during various hospital assessments performed at various poststroke time points. LMW cfDNA was detected at relatively high levels 24 hours after acute onset of ischemia in the brain but had not been seen at later time points. These findings are intriguing and may aid in post-hoc differentiation of acute ischemic strokes from "missed," false-negative cases, and from more or less common stroke over-diagnosis, also known as "stroke mimics" (Liberman \& Prabhakaram, 2017).

Interestingly, after combining the cohort of controls, significant inter-individual variation in plasma LMW cfDNA levels was observed. This variation points at possible physiological fluctuations in plasma LMW cfDNA levels, which may reflect the overall state of the body's response to intrinsic and extrinsic stress, the importance of taking into account individual baseline levels of plasma cfDNA, and the necessity of longitudinal assaying of plasma cfDNA in healthy cohorts.

\section{Ethical Considerations}

\section{Compliance with ethical guidelines}

All ethical principles are considered in this article. The participants were informed of the purpose of the research and its implementation stages. They were also assured about the confidentiality of their information and were free to leave the study whenever they wished, and if desired, the research results would be available to them.

\section{Funding}

The study partly was financially supported by the Government of Russia (Grant No.: RFMEFI58117X0020).

\section{Authors' contributions}

Conceptualization: Vladimir Bespalov; Methodology: Irina Vasilyeva; Investigations: Voznyuk Igor and Irina Vasilyeva; Writing-original draft: Ancha Baranova; Writing - review and editing: Irina Vasilyeva; Funding acquisition: Denis Baranenko, Resources: Vladimir Bespalov, Denis Baranenko; Supervision: Vladimir Bespalov and Igor Voznyuk.

\section{Conflict of interest}

The authors declared no conflict of interest.

\section{References}

Amani, H., Habibey, R., Hajmiresmail, S. J., Latifi, Sh., PazokiToroudi, H. R., \& Akhavan, O. (2017). Antioxidant nanomaterials in advanced diagnoses and treatments of ischemia reperfusion injuries. Journal of Materials Chemistry B, 5(48), 9452-76. [DOI:10.1039/C7TB01689A] [PMID]

Boyko, M., Ohayon, Sh., Goldsmith, T., Douvdevani, A., Gruenbaum, B. F., \& Melamed, I., et al. (2011). Cell-free DNA--a marker to predict ischemic brain damage in a rat stroke experimental model. Journal of Neurosurgical Anesthesiology, 23(3) 222-8. [DOI:10.1097/ANA.0b013e31821b536a] [PMID]

Bustamante, A., Simats, A., Vilar-Bergua, A., García-Berrocoso, T., \& Montaner, J. (2016). Blood/brain biomarkers of inflammation after stroke and their association with outcome: From C-reactive protein to damage-associated molecular patterns. Neurotherapeutics, 13(4), 671-84. [DOI:10.1007/s13311-0160470-2] [PMID] [PMCID]

Choi, J. H., \& Pile-Spellman, J. (2018). Reperfusion changes after stroke and practical approaches for neuroprotection. Neuro imaging Clinics of North America, 28(4), 663-82. [DOI:10.1016/j. nic.2018.06.008] [PMID]

Dieplinger, B., Bocksrucker, C., Egger, M., Eggers, C., Haltmayer, M., \& Mueller, T. (2017). Prognostic value of inflammatory and cardiovascular biomarkers for prediction of 90-day all-cause mortality after acute ischemic stroke-results from the Linz stroke unit study. Clinical Chemistry, 63(6), 1101-9. [DOI:10.1373/clinchem.2016.269969] [PMID]

Dilek, A., Alacam, H., Ulger, F., Bedir, A., Ulus, A., \& Murat, N. et al. (2013). Comparison of predictive powers of S100B and cell-free plasma DNA values in intensive care unit patients with intracranial hemorrhage. Journal of Critical Care, 28(5), 883.e1-7. [DOI:10.1016/j.jcrc.2013.03.005] [PMID]

Ermakov, A. V., Konkova, M. S., Kostyuk, S. V., Izevskaya, V. L., Baranova, A., \& Veiko, N. N. (2013). Oxidized extracellular DNA as a stress signal in human cells. Oxidative Medicine and 
Cellular Longevity, 2013, 649747. [DOI:10.1155/2013/649747] [PMID] [PMCID]

Geiger, S., Holdenrieder, S., Stieber, P., Hamann, G. F., Bruening, R., \& Ma, J., et al. (2006). Nucleosomes in serum of patients with early cerebral stroke. Cerebrovascular Diseases, 21(1-2), 32-7. [DOI:10.1159/000089591] [PMID]

Glebova, K., Veiko, N., Kostyuk, S., Izhevskaya, V., \& Baranova, A. (2015). Oxidized extracellular DNA as a stress signal that may modify response to anticancer therapy. Cancer Letters, 356(1), 22-33. [DOI:10.1016/j.canlet.2013.09.005] [PMID]

Go, A. S., Mozaffarian, D., Roger, V. L., Benjamin, E. J., Berry, J. D., \& Borden, W. B., et al. (2013). Heart disease and stroke statistics--2013 update: A report from the American Heart Association Circulation, 127(1), e6-245. [DOI:10.1161/CIR.0b013e31828124ad] [PMID] [PMCID]

Hefeneider, S. H., Cornell, K. A., Brown, L. E., Bakke, A. C., McCoy, S. L., \& Bennett, R. M. (1992). Nucleosomes and DNA bind to specific cell-surface molecules on murine cells and induce cytokine production. Clinical Immunology and Immunopathology, 63(3), 245-51. [DOI:10.1016/0090-1229(92)90229-H]

Lam, N. Y. L., Rainer, T. H., Wong, L. K. S., Lam, W., \& Lo, Y. M. D. (2006). Plasma DNA as a prognostic marker for stroke patients with negative neuroimaging within the first $24 \mathrm{~h}$ of symptom onset. Resuscitation, 68(1), 71-8. [DOI:10.1016/j.resuscitation.2005.05.021] [PMID]

Liberman, A. L., \& Prabhakaran, Sh. (2017). Stroke chameleons and stroke mimics in the emergency department. Current Neurology and Neuroscience Reports, 17(2), 15. [DOI:10.1007/s11910017-0727-0] [PMID]

Mackay, J., \& Mensah, G., (2004). The atlas of heart disease and stroke. Retrieved from https://www.who.int/cardiovascular_diseases/ resources/atlas/en/

Mittra, I., Nair, N. K., \& Mishra, P. K. (2012). Nucleic acids in circulation: Are they harmful to the host? Journal of Biosciences, 37(2), 30112. [DOI:10.1007/s12038-012-9192-8] [PMID]

O'Connell, G. C., Petrone, A. B., Tennant, C. S., Lucke-Wold, N., Kabbani, Y., \& Tarabishy, A. R., et al. (2017). Circulating extracellular DNA levels are acutely elevated in ischaemic stroke and associated with innate immune system activation. Brain Injury, 31(10), 1369-75. [DOI:10.1080/02699052.2017.1312018] [PMID]

Peters, D. L., \& Pretorius, P. J. (2011). Origin, translocation and destination of extracellular occurring DNA--a new paradigm in genetic behaviour. Clinica Chimica Acta, 412(11-12), 806-11. [DOI:10.1016/j. cca.2011.01.026] [PMID]

Rainer, T. H., Wong, L. K. S., Lam, W., Yuen, E., Lam, N. Y. L., \& Metreweli, C., et al. (2003). Prognostic use of circulating plasma nucleic acid concentrations in patients with acute stroke. Clinical Chemistry, 49(4), 562-9. [DOI:10.1373/49.4.562] [PMID]

Tsai, N. W., Lin, T. K., Chen, S. D., Chang, W. N., Wang, H. C., \& Yang, T. M., et al. (2011). The value of serial plasma nuclear and mitochondrial DNA levels in patients with acute ischemic stroke. Clinica Chimica Acta, 412(5-6), 476-9. [DOI:10.1016/j. cca.2010.11.036] [PMID]

Vasilyeva, I. N., Ivtchik, T. V., \& Voznyuk, I. A. (2010). Low-molecular-weight DNA of blood plasma as an indicator of pathological processes. In P. Gahan (Ed.), Circulating nucleic acids in plasma and serum (pp. 165-170). Dordrecht: Springer. [DOI:10.1007/978-90481-9382-0_23]
Zhivotosky, B., \& Orrenius, S. (2001). Assessment of apoptosis and necrosis by DNA fragmentation and morphological criteria. Current Protocols in Cell Biology, 12(1), 18.3.1-23. [DOI:10.1002/0471143030. cb1803s12] [PMID] 